\title{
Design of a Nanoscale, CMOS-Integrable, Thermal-Guiding Structure for Boolean-Logic and Neuromorphic Computation DOI:
}

10.1021/acsami.6b10667

\section{Document Version}

Accepted author manuscript

Link to publication record in Manchester Research Explorer

Citation for published version (APA):

Loke, D., Skelton, J. M., Chong, T. C., \& Elliott, S. R. (2016). Design of a Nanoscale, CMOS-Integrable, ThermalGuiding Structure for Boolean-Logic and Neuromorphic Computation. ACS Applied Materials and Interfaces, 8(50), 34530-34536. https://doi.org/10.1021/acsami.6b10667

\section{Published in:}

ACS Applied Materials and Interfaces

\section{Citing this paper}

Please note that where the full-text provided on Manchester Research Explorer is the Author Accepted Manuscript or Proof version this may differ from the final Published version. If citing, it is advised that you check and use the publisher's definitive version.

\section{General rights}

Copyright and moral rights for the publications made accessible in the Research Explorer are retained by the authors and/or other copyright owners and it is a condition of accessing publications that users recognise and abide by the legal requirements associated with these rights.

\section{Takedown policy}

If you believe that this document breaches copyright please refer to the University of Manchester's Takedown Procedures [http://man.ac.uk/04Y6Bo] or contact uml.scholarlycommunications@manchester.ac.uk providing relevant details, so we can investigate your claim.

\section{OPEN ACCESS}




\title{
Design Of A Nanoscale, CMOS-Integrable, Thermal-Guiding Structure For Boolean-Logic And Neuromorphic Computation
}

Desmond Loke, ${ }^{1, *}$ Jonathan M. Skelton, ${ }^{2}$ Tow-Chong Chong, ${ }^{1}$ Stephen R. Elliott ${ }^{3, *}$

${ }^{1}$ Science Faculty, Singapore University of Technology and Design, 8 Somapah Road, Singapore 487372, Singapore

${ }^{2}$ Department of Chemistry, University of Bath, Claverton Down, Bath BA2 7AY, UK

${ }^{3}$ Department of Chemistry, University of Cambridge, Lensfield Road, Cambridge CB2 1EW, UK

Email: desmond_loke@sutd.edu.sg, sre1@cam.ac.uk

Keywords: FEM Simulations, Thermal Control, Switches, Metamaterials, Computing

\begin{abstract}
One of the requirements for achieving faster CMOS electronics is to mitigate the unacceptably large chip areas required to steer heat away from or, more recently, towards the critical nodes of state-of-the-art devices. Thermal-guiding (TG) structures can efficiently direct heat by 'meta-materials' engineering; however, some key aspects of the behavior of these systems are not fully understood. Here, we demonstrate control of the thermal-diffusion properties of TG structures by using nanometer-scale, CMOS-integrable, graphene-on-silica (GOS) stacked materials through finite-element methods (FEM) simulations. It has been shown that it is possible to implement novel, controllable, thermally-based Boolean-logic and spike-timingdependent plasticity (STDP) operations for advanced (neuromorphic) computing applications using such thermal-guide architectures.
\end{abstract}

\section{Introduction}

The ever-increasing demand for faster CMOS electronics, optoelectronics and photonic devices has driven a widespread search for next-generation thermal-guiding (TG) structures. TG structures, based on the selective guiding of thermal diffusion around, as well as into, a target 
region of a TG matrix, ${ }^{1,2}$ are generally capable of not only steering heat away from critical nodes of integrated circuits (or silicon/chalcogenide devices) to construct higher density, and smaller, transistor devices, ${ }^{3,4}$ but also, more recently, able to direct heat towards key regions of silicon (or chalcogenide) devices to perform multiple, advanced circuit functions, such as Boolean-logic computations and brain-like (neuromorphic) computing. ${ }^{5,6}$ However, these TG systems are usually large; for example, in integrated circuits, TG structures in the form of heat spreaders and heat sinks are typically on the order of square millimeters, ${ }^{7,8}$ while in silicon/chalcogenide devices, TG structures are usually comprised of several hundred-nanometers-thick insulating layers. ${ }^{9,10}$. A difficulty arises from the trade-off between increasing (or reducing) the rate of heat transfer, and, at the same time, decreasing the contact area to avoid the challenges of integrating these architectures into high-density CMOS circuits. ${ }^{11}$

Recent efforts to overcome these limitations have been focused on inserting different materials and patterns in heat-sink and insulating-layer configurations to achieve more compact TG structures, for example, rings of polydimethylsiloxane (PDMS) in copper plates and sheets of fullerene $\mathrm{C}_{60}$ in silica films. ${ }^{12,13}$ However, some key aspects of the behavior of such TG systems remain unclear, which limits our ability to assess the ultimate down-scaling and performance of this technology. First, a related aspect concerns what the materials of TG structures could be, so as to prevent incompatibility with CMOS processing, and also how TG structures could be tuned to achieve multiple functions to increase the number of operations performed by a system. The most important consideration, perhaps, is how the thermal diffusion could be 'engineered' by TG structures to generate thermal-invisibility, as well as thermal-trapping, regions on the order of nanometer length scales. Unambiguous experimental information on these issues can be very 
difficult to obtain because of the high manufacturing costs involved and the small length scales of actual TG architectures. ${ }^{14-16}$ Hence, we have used simulations in the first instance to demonstrate proof of concept of our approach.

Despite efforts to obtain a better understanding through modeling or numerical calculations performed on TG structures, TG effects, and especially thermal-invisibility or socalled 'cloaking' phenomena upon structural down-sizing, are still not understood. ${ }^{17-19}$ Although several aspects of TG systems have been investigated using numerical-calculation methods, very few studies on the actual CMOS environment have yet been done. ${ }^{20-23}$ A possible reason for this might be due to the appreciable difficulties in finding CMOS-integrable materials to enable TG to take place in silico. Switchable TG structures have been demonstrated using thermal'pumping' processes that transport heat flux from one side to the other side of a system via thermoelectric components controlled by applied electric voltages. ${ }^{24}$ However, this heat pumping is a high flow-rate process, and this method requires transferring heat from the heat absorber to the heat emitter via a copper block with a size on the order of millimeters, due to the contradictory nature of increasing heat transfer while reducing contact area. ${ }^{11}$

Here, we demonstrate control of the thermal-diffusion properties of TG structures by using nanometer-scale, CMOS-integrable, stacked materials through finite-element-method (FEM) simulations. Such thermal-guide structures could be harnessed for applications in Boolean-logic and neuromorphic computations. These were achieved using TG structures made from graphene-on-silica (GOS) stacked materials. Graphene has been previously demonstrated to be an excellent inorganic template for achieving high thermal conduction parallel to the layers, 
while silica has been shown to be a good dielectric template for realizing low heat conductance. ${ }^{25,26}$ Additionally, the thermal contact resistance between graphene and silica at their interface can be lower than the contact resistance required in CMOS circuits, ${ }^{27}$ while thin graphene and silica films can be patterned and deposited using CMOS-based procedures. ${ }^{28,29}$ An ultrathin GOS-integrable TG structure was simulated, which could be used for multiple controllable thermal-switching operations, such as a complete set of Boolean logic computations, and the entire characteristics of spike-timing-dependent plasticity (STDP) in neuromorphic circuits. ${ }^{3,4}$ This approach avoids the necessity of having to use large chip areas by using embedded thermal-guide heat-conduction kinetics, rather than space-consuming thermalpumping dynamics. We further demonstrate that, by varying the width, diameter, period number, spacer width and also the delay timing for the GOS thermal-guide architectures, the thermaldiffusion properties of structures could be altered. Transient simulations revealed the ability of GOS architectures for guiding heat diffusion, which is observed to be the key to enhancing the thermal-switching performance.

\section{Methods}

We first investigate the thermal-diffusion properties of TG structures by carrying out FEM simulations on two-dimensional ( $x y$-plane) models of a series of one and two 2 nm-wide thermal-guide rings embedded in the archetypal CMOS host matrix, Si, using the ANSYS code. Possible design, fabrication and measurement details of the ring structures are given in Supporting Methods (see also Supporting Figures S1-3). Some of the simulations were of the 'swap-and-run' type, whereby the arrangement of the thermal guides was first systematically varied, and the host boundary conditions were set to $400 \mathrm{~K}$ at the left boundary and $300 \mathrm{~K}$ at the 
right boundary (see Supporting Methods). Steady-state simulations were performed and the material properties were kept constant. In order to assess the effects of the thermal guides on the host structure, two guide materials were utilized, viz. silica and graphene. Graphene contains a single layer, or a few layers, of $\mathrm{sp}^{2}$-bonded $\mathrm{C}$ atoms arranged in a honeycomb lattice, while silica consists of a connected network of tetrahedra of four $\mathrm{O}$ atoms surrounding a central $\mathrm{Si}$ atom. ${ }^{30,31}$ Due to the phenomenon of lower phonon scattering as compared to silica, graphene shows a higher in-plane thermal conductivity, ${ }^{25,26}$ which, we predict, should promote the guiding of thermal diffusion, e.g. in a ring enclosing a region.

\section{Results and Discussion}

Using a temperature-profile (TP) analysis, Si matrices, without thermal guides and with the above thermal boundary conditions, showed only non-uniform temperature distributions across the entire structure, in marked contrast to the silica-ring thermal-guide models, which exhibited rather uniform temperature profiles inside the rings, and non-uniform temperature distributions outside the rings, viz. ranges of $300-400 \mathrm{~K}$ vs $344-355 \mathrm{~K}$ and $300-400 \mathrm{~K}$, respectively (Figure 1a). Silica and silica-on-graphene thermal-guide rings created convergent patterns of temperature profiles centered on the rings, whereas graphene thermal-guide rings produced a divergent pattern (Figure 1a). However, graphene-on-silica (GOS) thermal-guide rings acted as near-perfect thermal-cloaking structures, where the temperature inside the guide ring stayed low and uniform. Outside the ring, the temperature profile closely resembled that of the bare $\mathrm{Si}$ structure without thermal guides. Additionally, GOS thermal-guide models displayed lower peak temperatures inside the rings than did the pure Si models without thermal guides, viz. 354K vs $383 \mathrm{~K}$, respectively (see the histogram in Figure 1a; bottom right) and, along with equivalent 
thermal-guide modeling (Figure 1b), this offers an alternative strategy to guide heat away from key regions of CMOS devices. Similar thermal-cloaking phenomena were also observed with varying structural boundary conditions, model scales, matrix dimensions, dimension space $(z-$ axis), as well as temperature steps (viz. 8 and 11K) (Figure 1c, see also Supporting Figures S47), in agreement with the findings shown in Figure 1a.

We next examine the thermal-diffusion properties of TG structures for alternative materials by studying the thermal signatures of GOS thermal guides for chalcogenides. In chalcogenide phase-change random-access memory (PCRAM) devices, the PCRAM materials are prone to spontaneous (thermal) crystallization of the unstable amorphous phase, thus limiting overall long-term data retention. ${ }^{32}$ Additionally, PCRAM materials have one of the lowest thermal conductivities of any known material, ${ }^{33}$ which makes it very difficult to shield PCRAM devices from thermal diffusion. We modeled Si matrices containing a disc of a conventional PCRAM material, $\mathrm{Ge}_{2} \mathrm{Sb}_{2} \mathrm{Te}_{5}$ (GST), surrounded by a Si-spacer and a GOS-ring thermal guide. Si:GST matrices without thermal-guide rings showed convergent patterns of temperature profiles in the GST region, with a large perturbation of the temperature profile in the surrounding matrix (Figure 1d, top left). In contrast, ring thermal guides around the GST region produced much less perturbation to the temperature profile in the surrounding Si matrix (Figure 1d). The 8nm-wide thermal guides produced a more uniform temperature distribution (or thermal-cloaking patterns) inside the rings relative to the case for 4nm-wide thermal guides, viz. $333-366 \mathrm{~K}$ vs $344-355 \mathrm{~K}$, respectively (Figure 1d). Generally, PCRAM materials can be divided into two groups, depending on their crystallization mechanism, viz. nucleation-dominated or growth-dominated (e.g. GST vs $\mathrm{Ag}_{8} \mathrm{In}_{14} \mathrm{Sb}_{55} \mathrm{Te}_{23}$ (AIST)). ${ }^{34}$ In addition, AIST shows a higher thermal conductivity 
than does GST (see Methods). An almost identical thermal-cloaking effect was also observed for matrices with AIST discs (see also Supporting Figure S8), in agreement with the results shown in Figures 1a,c, suggesting that the ability of thermal guides for cloaking these materials is universal to all PCRAM materials, and that the thermal-cloaking phenomenon is robust to not only structural variations (i.e. boundary settings, model scales, matrix size), but also material alterations.

To understand how TG structures were capable of producing various temperature distributions or thermal-cloaking profiles under steady-state simulation conditions, transient simulations of silica- and graphene-based thermal guides in $\mathrm{Si}$ matrices were conducted at various time scales. Consistent with the prediction (as well as the findings in Figure 1), we found that the Si-core materials showed slower thermal diffusion than the non-Si-core materials, while graphene exhibited faster thermal diffusion relative to silica through a so-called 'relative thermal diffusional' behavior (Figure 2a). Thermal-guide rings employed by other research groups often show a dependence of thermal-diffusion properties upon size. ${ }^{35}$ In addition, we studied the effects of the size of thermal guides by performing simulations using GOS architectures with different ring-period configurations, or times between 0.01 and $1 \mathrm{~ns}$, as well as in steady state. Similar to other thermal-guide rings, the TG models showed higher peak temperatures inside the rings, the smaller the number of periods of the rings (Figure 2b). The same result was also observed for a decrease in the width of the thermal-guide rings (see also Supporting Figure S10), which suggests that the strength of thermal cloaking can be controlled by varying the sizes of the thermal guides. In contrast, the TG systems displayed lower peak temperatures within the rings, the smaller the diameter of the rings, meaning that an improvement of the thermal properties of 
TG structures via thermal cloaking can be achieved with device down-sizing. We note that the cloaking times required to achieve a larger contrast of peak core temperatures between pure $\mathrm{Si}$ models and GOS thermal-guide models can be shorter than the times required to process information in conventional CMOS devices. We believe that, through additional selection and material engineering, improved target system parameters, including thermal-cloaking times, can be achieved.

Now, we investigate the thermal-diffusion properties of TG structures of varying structural designs by studying the thermal signatures of GOS thermal guides in the form of strips. GOS thermal guides, comprising two 4nm-wide guide strips, were used to construct a series of thermal-guide configurations in the Si host matrices, viz. 'line', 'L' and 'U'. Possible design, fabrication and measurement details of the structures are similar to those utilized for the thermalguide rings (see Supporting Methods, find also Supporting Figures S11,12). Boundary conditions were set to be $400 \mathrm{~K}$ at the start of the flux-guide boundary and $300 \mathrm{~K}$ at the end of the flux-guide boundary, and the simulation time was chosen to be 4 or 5 ps. TG systems can also show thermalconcentration effects by confining heat sources within guide strips of fan-like configurations. ${ }^{36}$ The non-guided models showed non-uniform temperature distributions throughout the entire models, in contrast to the TG models, which exhibited uniform temperature spreads outside the guides and confined heat transport, associated with non-uniform temperature distributions inside the guides. This implies that thermal-concentration effects can persist for varying TG configurations, viz. $300-400 \mathrm{~K}$ vs $300 \mathrm{~K}$ and $300-400 \mathrm{~K}$, respectively (Figure $3 \mathrm{a}$ ). The thermalguide models also showed heat confinement along the $z$-direction (see also Supporting Figure S13). As expected, the non-guided systems displayed higher peak temperatures at the boundaries 
relative to the thermal-guided systems, viz. $307 \mathrm{~K}$ vs $302 \mathrm{~K}$, respectively (see also Supporting Figure S14).

To investigate applications of the thermal-diffusion properties of TG structures for varying schemes of excitation, we were further able to perform thermally-based Boolean-logic computations on a single structure by comparing two configurations of 4-way crossed GOSguided models, i.e. thermally non-biased and biased, based on our previous excitation scheme for chalcogenide devices. ${ }^{37}$ The possible design, fabrication, and measurement details of these structures are almost identical to those for the thermal-guide rings (Supporting Methods; see also Supporting Figures 11,12). Details of possible bias settings are also described in Supporting Methods (see also Suppporting Figure S15). With the same principles utilized in Figure 3a, the boundary conditions were set to be $300 \mathrm{~K}$ or $400 \mathrm{~K}$ at the left and bottom flux-guide boundaries for the inputs 0 or 1 , and $400 \mathrm{~K}$ at the top flux-guide boundary for the bias signals. The simulation time was kept constant at $4 \mathrm{ps}$, and the resulting temperatures at the flux-guide intersections were calculated and referenced for binary-state attribution $(0: T<330 \mathrm{~K} ; 1: T>$ 330K). Upon thermal excitation, the non-biased models showed the output values $0-0-0-1$ for the inputs 00-01-10-11 (Figures 3b,c). In contrast, the biased models exhibited the output results 0-11-1 for the inputs 00-01-10-11, viz. the logic operations AND: 300-319-319-338K, and OR: 320339-339-359K, in agreement with the results shown in Figure 3a (as well as the operations of other research groups ${ }^{4}$ ), indicating that the thermal-concentration effects can be preserved for alternate excitation schemes. Supporting Methods describe the details of possible re-usability of the outputs (see also Supporting Figure S16). Furthermore, the boundary conditions were set to be $300 \mathrm{~K}$ or $400 \mathrm{~K}$ at only the left flux-guide boundary for the inputs 0 or 1 , and $400 \mathrm{~K}$ at both top 
and bottom flux-guide boundaries for the bias signals. As anticipated, the non-biased models displayed the output logical outcome ' 0 ' for the input ' 1 ', as compared to the biased models, which showed the output signal '1' for the input '0'; viz. NOT: 339, 319K (Figures 3d,e). A similar result was also observed for steady-state simulations (see also Supporting Figure S17). Structures with a higher thermal concentration can also yield lower surrounding temperatures to reduce the thermal disturbance between adjacent systems. ${ }^{36}$ Although both non-guided and guided matrices showed the same results, the non-guided systems exhibited higher peak temperatures at the matrix boundary than did the guided systems. This also means that the thermal-guided structures can minimize thermal cross-talk during information processing (see also Supporting Figure S18). It should be noted that these multiple computations on a single structure could not simply be achieved by previous alternative short-circuit-based thermal transistors, ${ }^{4}$ which only provide one of the 2 inputs required, insufficient to realize 4 input combinations and 1 set of output values (for one operation), in contrast to the present additivebiased-based logic computing structure which offers all 2 inputs assisted by biasing to achieve 8 input combinations and 2 sets of output values (for two operations). Additionally, these computations could not be easily realized by previous thermal-pumping methods (on a scale of the order of nanometers), which require a metal block with a size on the order of millimeters. ${ }^{24}$

Although not central to this study, other interesting aspects of the dependence of thermaldiffusion properties on the delay of excitation timings are now described through an application to neuromorphic computing. The main components in neuromorphic circuits are devices that can control the strength of connections between other components in response to varying input signals, mimicking the functions of biological synapses. ${ }^{3}$ Exposure to an input signal yields a 
temporary variation in synaptic weight, which rapidly decays back to the initial value, a behavior known as short-term plasticity (STP), while repeated exposure to similar input patterns results in more permanent weight variations, leading to gradual increases or decreases, defined as longterm potentiation (LTP) and long-term depression (LTD), respectively. These processes form the basis of so-called Hebbian learning, a scheme commonly used to interpret the response of animal neurons to stimuli. ${ }^{38}$ In this model, each synapse receives input from two sources, i.e. a presynaptic and a postsynaptic neuron. Correlated activity spikes on these inputs leads to LTP or LTD, depending on the relative time delay between them, whereas uncorrelated inputs cause only short-lived variations through STP. This relationship between signal correlation and evolution of synaptic weight is often termed 'spike-timing-dependent plasticity' (STDP).

Our crossed four-way GOS thermal-guided models were exposed to varying bias settings and spike-timing delays. The boundary conditions were set to a series of $400 \mathrm{~K}$ pulses at the left flux-guide boundary for the prespike pulses, and a single $400 \mathrm{~K}$ pulse at the bottom flux-guide boundary for the postspike pulse. The durations of the prespike pulses were chosen to be 6.0, 3.0 and 1.0ps, and the time intervals between the prespike pulses were kept constant at 94.0, 97.0 and 99.0ps. The duration of the postspike pulse was kept the same at $6.0 \mathrm{ps}$, and the resulting temperatures at the flux-guide intersection after the application of each prespike pulse were analyzed. The changes in synaptic weight, $\Delta w$, which is given by the difference between the peak intersection temperature $T_{\mathrm{p}}$, for each spike-timing delay $t_{\mathrm{d}}$, and the reference temperature $T_{\text {ref, }}$, were recorded ( $\Delta w=T_{\mathrm{p}}-T_{\text {ref: }} T_{\text {ref }}=370 \mathrm{~K}$ for $\Delta t_{\mathrm{d}}<0 \mathrm{~s} ; T_{\text {ref }}=330 \mathrm{~K}$ for $\left.\Delta t_{\mathrm{d}}>0 \mathrm{~s}\right)$. Using thermal excitations, the non-biased prespike-after-postspike models showed a lower negative change of synaptic weights with an increase in the negative spike-timing delays, while the biased prespike- 
before-postspike models exhibited a lower positive change of synaptic weights with an increase in the positive spike-timing delay, viz. depression: -100ps, -200ps, -300ps; -41.9, -31.0, -26.8, and potentiation: 100ps, 200ps, 300ps; 35.4, 29.1, 12.6 (Figure 4), in parallel with results in Figure 3, and suggest that the magnitude of the thermal concentration can be altered by varying the delay of the excitation timings.

These simulation results suggest that controllable and advanced thermal-switching operations can be produced using thermal guiding with GOS structures. By using thermal guides with a stronger propensity for thermal diffusion, a systematic increase in the contrast of temperature variations could be achieved during switching.

\section{Conclusion}

In this simulational work, we have employed a graphene-on-silica (GOS) stacked material as a model thermal-transport guide to enable thermal cloaking, as well as thermal concentration, of a CMOS-integrable, ultrathin, embedded structure, and which also demonstrates controllable and advanced thermal-switching operations. Use of the simulational approach outlined here could potentially lead to the design of superior TG structures in the future by finding materials, for example, that cool nanometer-size regions for extended periods, or to much lower temperatures, and for the investigation of the effects of thermal guiding, for instance, with chevron-like patterns. This in silico simulation method, well suited to the study of the creation of CMOS-integrable TG configurations, should be a complementary, cost-effective, alternative technique to established experimental methods, involving system-level aspects of 
thermal cloaking and concentration, for the rapid screening of structures and materials for TGenhanced, site-specific, embedded cooling and heating solutions.

\section{Acknowledgements}

The authors thank W. J. Wang and R. Zhao for valuable discussions on the structural-simulation studies. We acknowledge financial support by the Engineering and Physical Sciences Research Council (UK). The FEM simulations were facilitated by the A*STAR Data Storage Institute computing facility. D. Loke thanks the SUTD Temasek Labs Seed Program for research support.

\section{Supporting Information}

Thermal-guide structures, ring thermal-guide design and fabrication, ring thermal-guide characterization, simulation procedures, thermal-guide structures for PCRAMs, strip thermalguide design and fabrication, bias settings and re-usability of outputs. 


\section{References}

1) Leonhardt, U. Applied Physics: Cloaking of Heat. Nature 2013, 498, 440-441.

2) Guenneau, S.; Amra, C.; Veynante, D. Transformation Thermodynamics: Cloaking and Concentrating Heat Flux. Opt. Express 2012, 20, 8207-8218.

3) Han, H.; Zhang, Y.; Wang, N.; Samani, M. K.; Ni, Y.; Mijbil, Z. Y.; Edwards, M.; Xiong, S.; Sääskilahti, K.; Murugesan, M.; Fu, Y. Functionalization Mediates Heat Transport in Graphene Nanoflakes. Nat. Commun. 2016, 7, 11281.

4) Nukala, P.; Lin, C. C.; Composto, R.; Agarwal, R. Ultralow-Power Switching via Defect Engineering in Germanium Telluride Phase-Change Memory Devices. Nat. Commun. 2016, 7, 10482.

5) Tuma, T.; Pantazi, A.; Le Gallo, M.; Sebastian, A.; Eleftheriou, E. Stochastic PhaseChange Neurons. Nat. Nanotechnol. 2016, 11, 693-699.

6) Wang, L.; Li, B. Thermal Logic Gates: Computation with Phonons. Phys. Rev. Lett. 2007, 99, 177208-1-4.

7) Chen, F.; Lei, D. Y. Experimental Realization of Extreme Heat Flux Concentration with Easy-to-Make Thermal Metamaterials. Sci. Rep. 2015, 5, 11552.

8) Narayana, S.; Sato, Y. Heat Flux Manipulation with Engineered Thermal Materials. Phys. Rev. Lett. 2012, 108, 214303-1-5.

9) Koelmans, W. W.; Sebastian, A.; Jonnalagadda; V. P.; Krebs, D.; Dellmann, L.; Eleftheriou, E. Projected Phase-Change Memory Devices. Nat. Commun. 2015, 6, 8181. 
10) Park, J. H.; Kim, H. Y.; Jang, G. S.; Seok, K. H.; Chae, H. J.; Lee, S. K.; Kiaee, Z.; Joo, S. K. Integrating Epitaxial-Like $\mathrm{Pb}(\mathrm{Zr}, \mathrm{Ti}) \mathrm{O}_{3}$ Thin-Film into Silicon for Next-Generation Ferroelectric Field-Effect Transistor. Sci. Rep. 2016, 6, 23189.

11) Lee, S. Optimum Design and Selection of Heat Sinks. Annu. IEEE Semicond. Therm. Meas. Manage. Symp. 1995, 48-54.

12) Schittny, R.; Kadic, M.; Guenneau, S.; Wegener, M. Experiments on Transformation Thermodynamics: Molding the Flow of Heat. Phys. Rev. Lett. 2013, 110, 195901-1-5.

13) Kim, C.; Suh, D. S.; Kim, K. H.; Kang, Y. S.; Lee, T. Y.; Khang, Y.; Cahill, D. G. Fullerene Thermal Insulation for Phase Change Memory. Appl. Phys. Lett. 2008, 92, 013109-1-3

14) Ma, Y.; Lan, L.; Jiang, W.; Sun, F.; He, S. A Transient Thermal Cloak Experimentally Realized Through a Rescaled Diffusion Equation with Anisotropic Thermal Diffusivity. NPG Asia Mater. 2013, 5, e73.

15) Han, T.; Bai, X.; Gao, D.; Thong, J. T.; Li, B.; Qiu, C. W. Experimental Demonstration of a Bilayer Thermal Cloak. Phys. Rev. Lett. 2014, 112, 054302-1-5.

16) Shen, L.; Zheng; B., Liu, Z.; Wang, Z.; Lin, S.; Dehdashti, S.; Li, E.; Chen, H. LargeScale-Far-Infrared Invisibility Cloak Hiding Object from Thermal Detection. Adv. Opt. Mater. 2015, 3, 1738-1742.

17) Fan, C. Z.; Gao, Y.; Huang, J. P. Shaped Graded Materials with an Apparent Negative Thermal Conductivity. Appl. Phys. Lett. 2008, 92, 251907-1-3.

18) Athanasopoulos, N.; Siakavellas, N. J. Heat Manipulation Using Highly Anisotropic Pitch-Based Carbon Fiber Composites. Adv. Eng. Mater. 2015, 17, 1494-1503. 
19) Chen, T., Weng, C. N.; Chen, J. S. Cloak for Curvilinearly Anisotropic Media in Conduction. Appl. Phys. Lett. 2008, 93, 114103-1-3.

20) Hu, R.; Wei, X.; Hu, J.; Luo, X. Local Heating Realization by Reverse Thermal Cloak. Sci. Rep. 2014, 4, 3600.

21) Han, T.; Yuan, T.; Li, B.; Qiu, C. W. Homogeneous Thermal Cloak with Constant Conductivity and Tunable Heat Localization. Sci. Rep. 2013, 3, 1593.

22) Dede, E. M.; Nomura, T.; Schmalenberg, P.; Lee, J. S. Heat Flux Cloaking, Focusing, and Reversal in Ultra-Thin Composites Considering Conduction-Convection Effects. Appl. Phys. Lett. 2013, 103, 063501-1-4.

23) Zhang, Y.; Xu, H.; Zhang, B. Design, Implementation, and Extension of Thermal Invisibility Cloaks. AIP Adv. 2015, 5, 053402-1-12.

24) Nguyen, D. M.; Xu, H.; Zhang, Y.; Zhang, B. Active Thermal Cloak. Appl. Phys. Lett. 2015, 107, 121901-1-4.

25) Balandin, A. A. Thermal Properties of Graphene and Nanostructured Carbon Materials. Nat. Mater. 2011, 10, 569-581.

26) Kleiner, M. B.; Kühn, S.; Weber, W. Thermal Conductivity Measurements of Thin Silicon Dioxide Films in Integrated Circuits. IEEE Trans. Electron Devices 1996, 43, 1602-1609.

27) Leong, W. S., Gong, H., Thong, J. T. Low-Contact-Resistance Graphene Devices with Nickel-Etched-Graphene Contacts. ACS Nano 2013, 8, 994-1001.

28) Jacob, M. V.; Rawat, R. S.; Ouyang, B.; Bazaka, K.; Kumar, D. S.; Taguchi, D.; Iwamoto, M.; Neupane, R.; Varghese, O. K. Catalyst-Free Plasma Enhanced Growth of Graphene from Sustainable Sources. Nano Lett. 2015, 15, 5702-5708. 
29) Han, L., Chen, Z. High-Quality Thin $\mathrm{SiO}_{2}$ Films Grown by Atomic Layer Deposition Using Tris (dimethylamino) Silane (TDMAS) and Ozone. ECS J. Solid State Sc. 2013, 2, N228-N236.

30) Meyer, J. C.; Geim, A. K.; Katsnelson, M. I.; Novoselov, K. S.; Booth, T. J.; Roth, S. The Structure of Suspended Graphene Sheets. Nature 2007, 446, 60-63.

31) Warren, B. E.; Biscoe, J. The Structure of Silica Glass by X-Ray Diffraction Studies. J. Am. Ceram. Soc. 1938, 21, 49-54.

32) Raoux, S.; Wuttig, M. Phase Change Materials: Science and Applications. Springer Science \& Business Media 2010.

33) Lyeo, H. K.; Cahill, D. G.; Lee, B. S.; Abelson, J. R.; Kwon, M. H.; Kim, K. B.; Bishop, S. G.; Cheong, B. K. Thermal Conductivity of Phase-Change Material $\mathrm{Ge}_{2} \mathrm{Sb}_{2} \mathrm{Te}_{5}$. Appl. Phys. Lett. 2006, 89, 151904-1-3.

34) Zhou, G. F. Materials Aspects in Phase Change Optical Recording. Mater. Sci. Eng. A 2001, 304, 73-80.

35) Xu, H.; Shi, X.; Gao, F.; Sun, H.; Zhang, B. Ultrathin Three-Dimensional Thermal Cloak. Phys. Rev. Lett. 2014, 112, 054301-1-5.

36) Han, T.; Bai, X.; Liu, D.; Gao, D.; Li, B.; Thong, J. T.; Qiu, C. W. Manipulating Steady Heat Conduction by Sensu-Shaped Thermal Metamaterials. Sci. Rep. 2015, 5, 10242.

37) Lee, T. H.; Loke, D.; Huang, K. J.; Wang, W. J.; Elliott, S. R. Tailoring TransientAmorphous States: Towards Fast and Power-Efficient Phase-Change Memory and Neuromorphic Computing. Adv. Mater. 2014, 26, 7493-7498. 
38) Kuzum, D.; Jeyasingh, R. G.; Lee, B.; Wong, H. S. P. Nanoelectronic Programmable Synapses Based on Phase Change Materials for Brain-Inspired Computing. Nano Lett. 2011, 12, 2179-2186. 


\section{Figures}

\section{Figure 1}
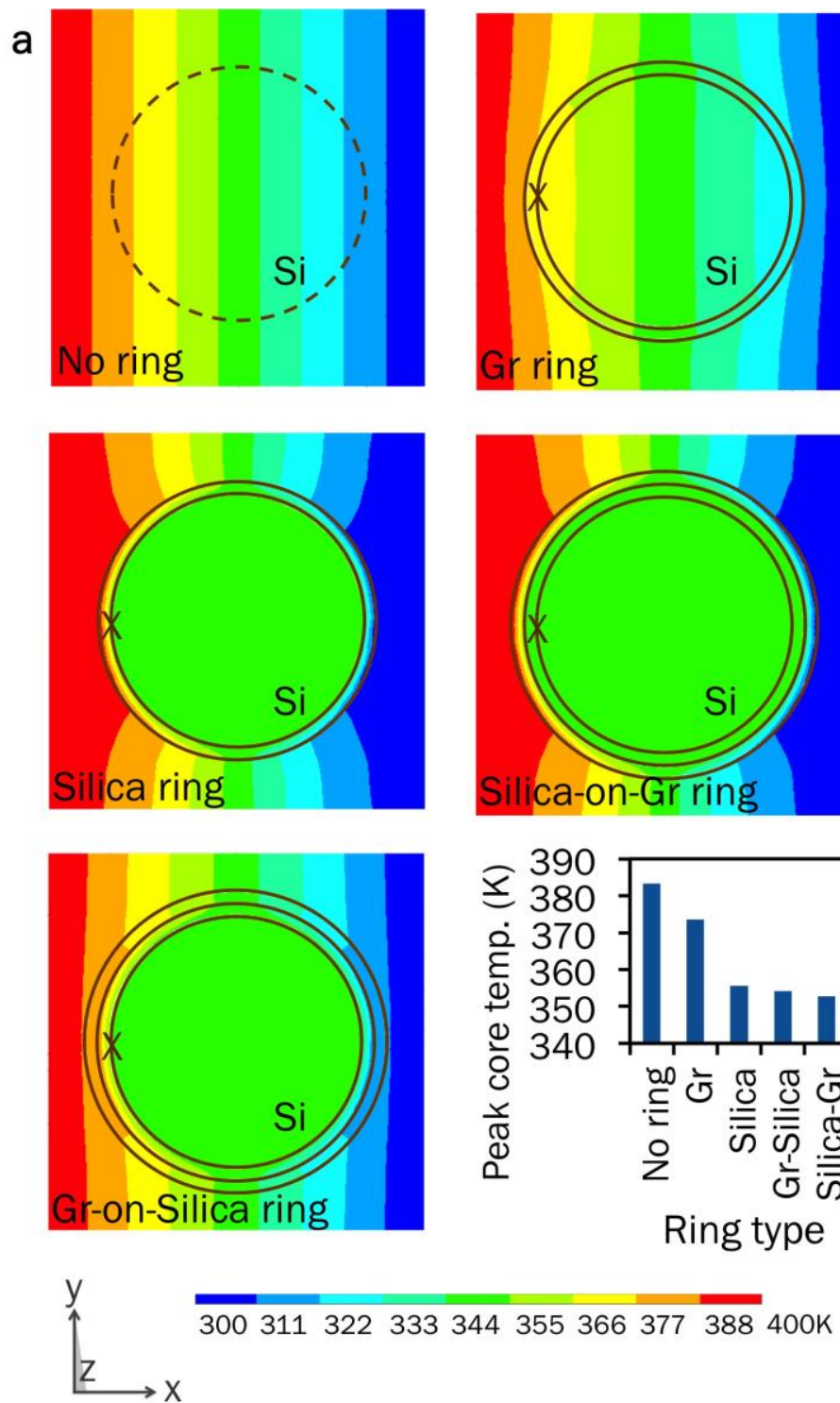

$\mathrm{Si}$ b
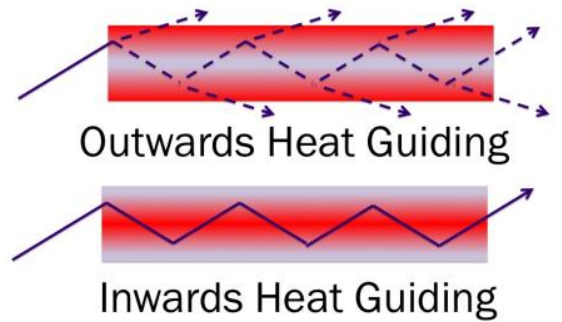

c
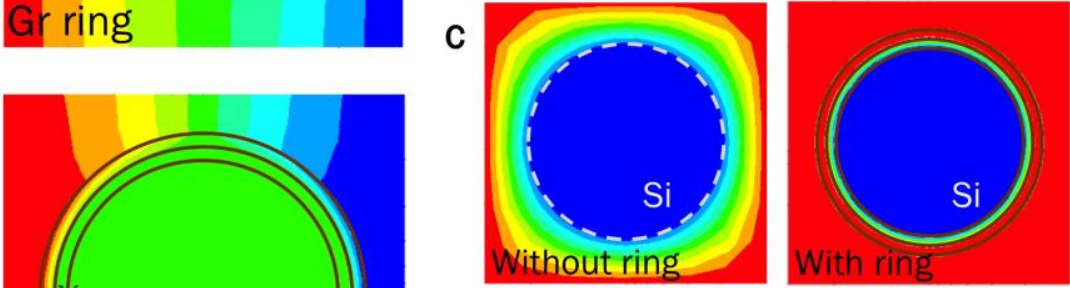

d
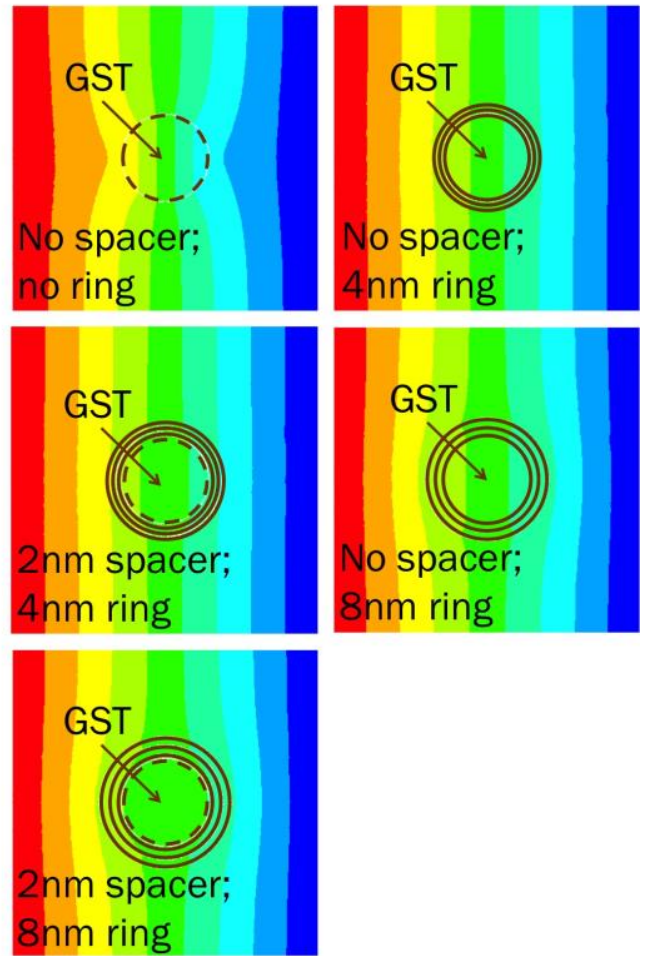
Figure 2

a
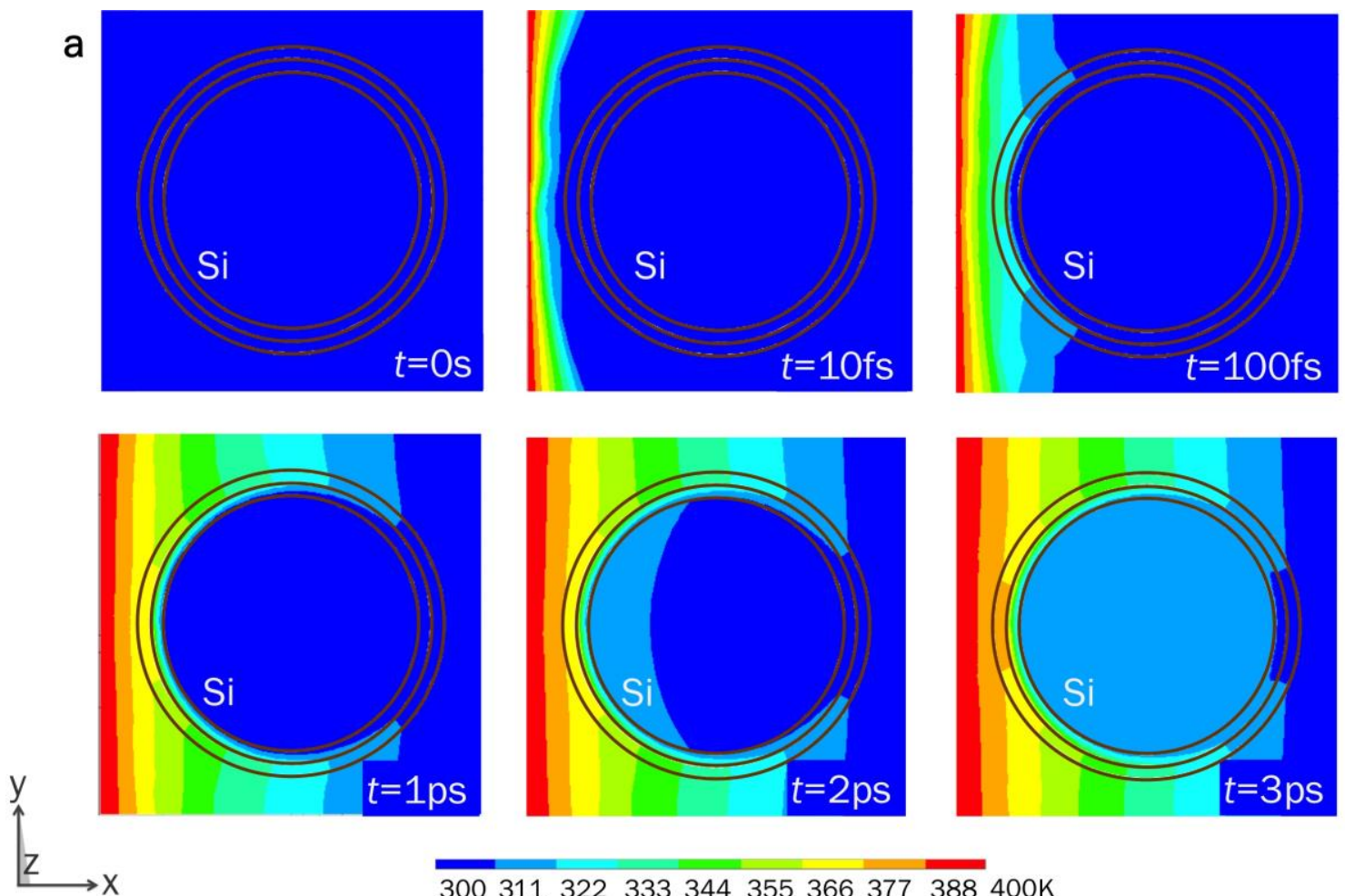

300311322333344355366377388 400K
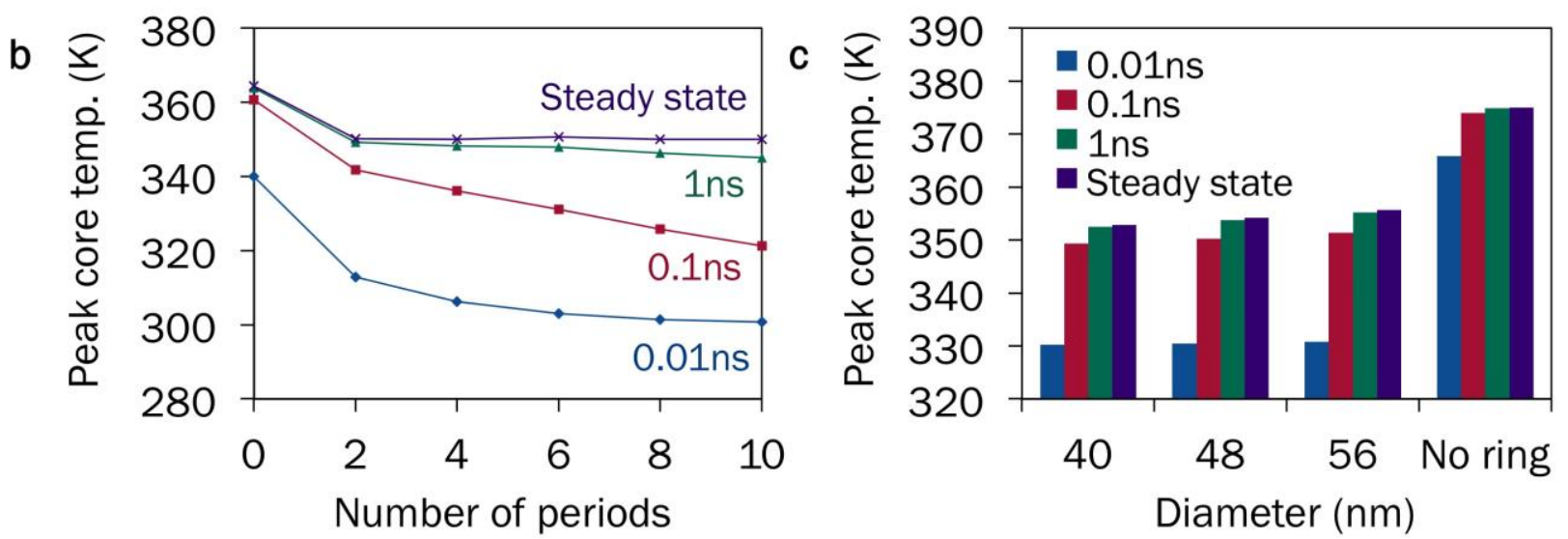
Figure 3
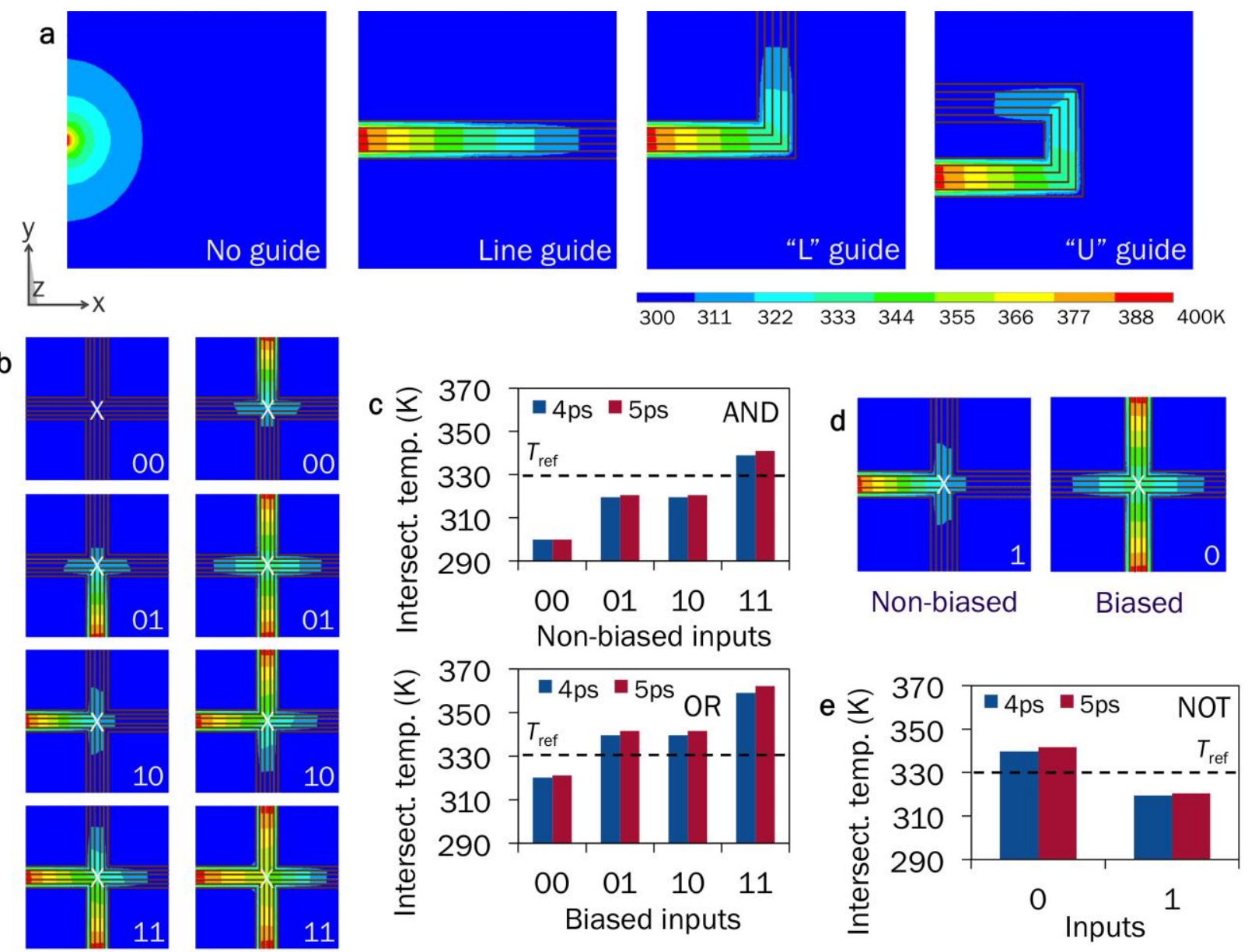

Non-biased

Biased 
Figure 4
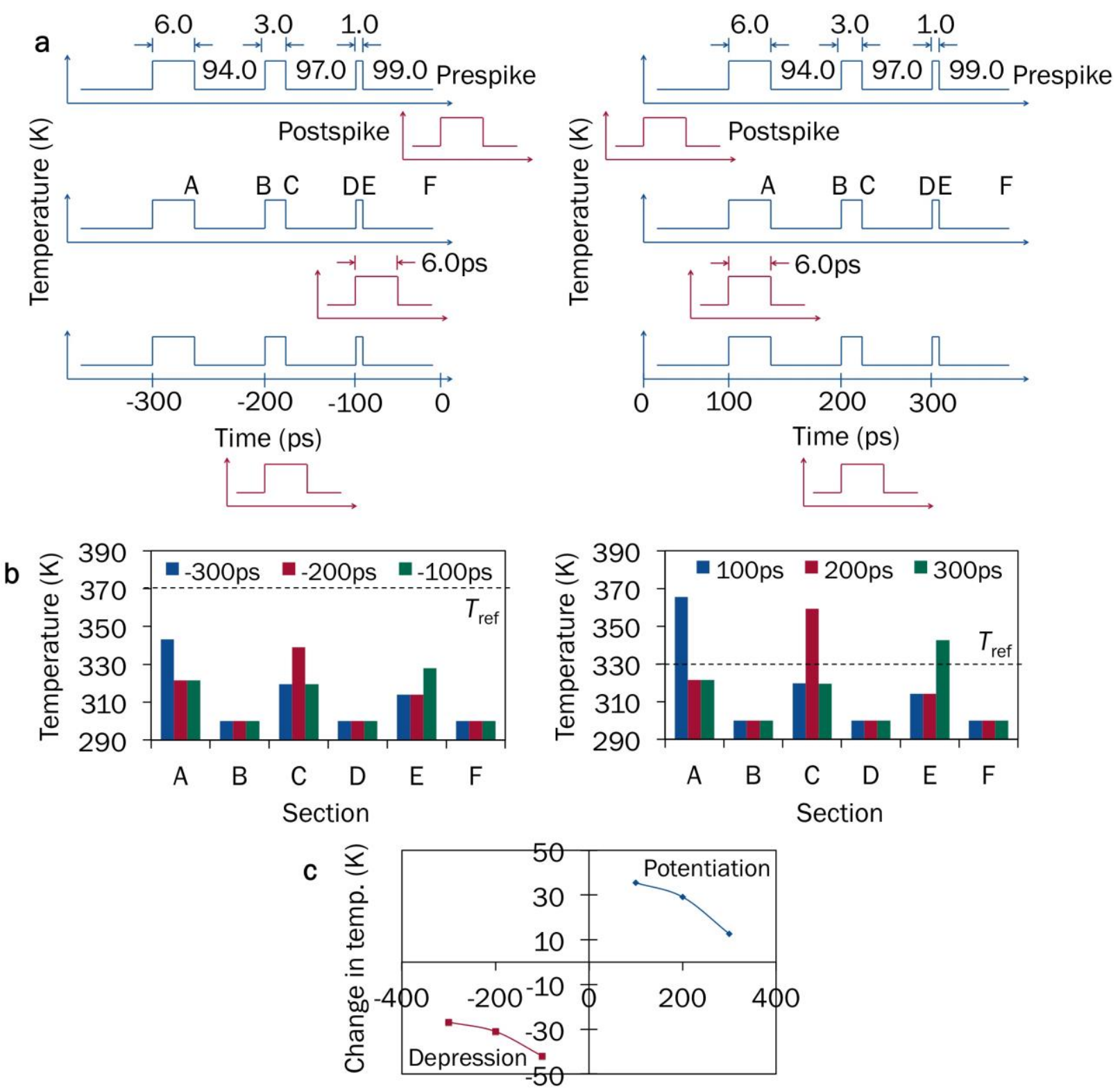

Time (ps) 


\section{Figure captions}

Figure 1. Heat shielding via thermal-diffusion guides. a, Steady-state temperature distributions of models of Si structures with and without silica and graphene thermal-diffusion rings. The symbol ' $\mathrm{X}$ ' marks the position where the peak temperature inside the ring was calculated. The dependence of the peak temperature inside a guide ring on the ring type employed for the models is also shown (bottom right). The boundary conditions were set to be $400 \mathrm{~K}$ at the left boundary and $300 \mathrm{~K}$ at the right boundary. The color coding for temperatures is shown at the bottom. b, Simple models showing thermal guides (in light purple) steering heat away from a region of columnar form, as well as trapping heat within the columnar region. c, Steady-state temperature profiles of Si structures with no guide ring and with a GOS guide ring: heat shielding for the boundary conditions set to $300 \mathrm{~K}$ inside the ring and $400 \mathrm{~K}$ at the $\mathrm{Si}$ structure boundaries. $\mathbf{d}$, Temperature profiles of structures containing a $\mathrm{Ge}_{2} \mathrm{Sb}_{2} \mathrm{Te}_{5}(\mathrm{GST})$ disc in a surrounding Si matrix without and with GOS-ring thermal guides with various widths and Sispacer rings. The rings contained a 2 or $4 \mathrm{~nm}$-wide ring of silica and a 2 or $4 \mathrm{~nm}$-wide ring of graphene, while the spacer comprised a $2 \mathrm{~nm}$-wide ring of $\mathrm{Si}$. The temperature boundary conditions were as in (a). The dimension of the matrix was kept constant at $60 \times 60 \mathrm{~nm}(\mathbf{a}, \mathbf{c})$ and $140 \times 140 \mathrm{~nm}(\mathbf{d})$. The diameter of the core of the matrix was held constant at $40 \mathrm{~nm}$. The width of each ring was kept constant at $2 \mathrm{~nm}$ for $(\mathbf{a}, \mathbf{c})$. All simulations were performed under steady-state conditions.

Figure 2. Time-dependent behavior of GOS thermal-diffusion guides. a, Temperature distributions of models of Si structures with GOS-ring thermal guides at varying times. b, Plots 
showing the peak temperature inside the thermal-guide ring, at $\mathrm{X}$, of models of GOS-ring thermal guides for a varying number of period of rings. c, Dependence of the peak temperature (at $\mathrm{X}$ ), within GOS thermal-guide rings in a $\mathrm{Si}$ matrix, on the diameter of the rings. The boundary conditions were set to be $400 \mathrm{~K}$ at the left boundary and $300 \mathrm{~K}$ at the right boundary. The dimension of the Si structure was kept constant at $60 \times 60 \mathrm{~nm}(\mathbf{a}), 140 \times 140 \mathrm{~nm}(\mathbf{b})$ and $80 \times 80 \mathrm{~nm}(\mathbf{c})$. The diameter of the central region inside the ring guides was held constant at $40 \mathrm{~nm}$, and the width of each ring was kept constant at $2 \mathrm{~nm}$. The temperature profiles of the models for ten periods of rings and at different times are also shown in Figure S9.

Figure 3. GOS thermal-diffusion-guide structural models for thermal-switching Booleanlogic operations. a, Temperature distributions for Si structures with GOS thermal-flux guides for various guide architectures. The boundary conditions were set to be $400 \mathrm{~K}$ at one end of the flux guide and $300 \mathrm{~K}$ at the other end. b, Temperature profiles of Si structures containing crossed four-way GOS thermal-diffusion guides with varied thermal inputs at the left and bottom fluxguide boundaries, with and without thermal biases at the top flux-guide boundary, used for Boolean-logic operations. c, Dependence of the temperature values at the intersection (marked ' $\mathrm{X}$ ') of the models shown in (a) used for AND and OR Boolean-logic computations. d, Temperature profiles in Si structures containing crossed four-way GOS thermal-diffusion guides with various thermal inputs at the left flux-guide boundary, and with and without thermal biases at both top and bottom flux-guide boundaries, for Boolean-logic operations. e, Plot showing the temperature values calculated at the intersection (marked ' $X$ ') of the models shown in (d), used for NOT Boolean-logic computation. The dimensions of the structures were kept constant at $140 \times 140 \mathrm{~nm}$, and the widths of the guides were held constant at $4 \mathrm{~nm}$. The boundary conditions 
were set to be $300 \mathrm{~K}$ or $400 \mathrm{~K}$ for the inputs 0 or 1 , and $300 \mathrm{~K}$ or $400 \mathrm{~K}$ for the no-bias and bias settings, respectively. The right flux-guide boundary was kept constant at $300 \mathrm{~K}$, and the reference temperature, used to discriminate logical outputs, was set at $330 \mathrm{~K}$. The simulation time was kept constant at 4 ps for (a), and 4 and 5 ps for (b-e).

Figure 4. Programming of GOS thermal-guide structural models for realizing thermallybased STDP-learning circuits. a, Schematic configurations illustrating the pre- and postspike pulse-excitation scheme for the structural models with varied spike-timing delays. b, Plots showing the temperature values for the structural model after each pulse excitation and rest interval for the prespike-after-postspike (left) and prespike-before-postspike (right) pulses. c, Change of temperature values for the structural model on 'depression' and 'potentiation' operations. The structural model employed is identical to the crossed four-way GOS thermaldiffusion guide configuration shown in Figure $3 \mathrm{~b}$. The prespike pulses were applied at the left flux-guide boundary, and the postspike pulse was delivered at the bottom flux-guide boundary. A thermal bias pulse was utilized during the prespike-before-postspike pulse excitation, and it was applied when both prespike and postspike pulses were applied. The prespike, postspike and bias pulses were kept constant at $400 \mathrm{~K}$, and the rest-interval temperature was held constant at $300 \mathrm{~K}$. The right flux-guide boundary was kept the same at $300 \mathrm{~K}$. The output temperature values were obtained at the intersection (' $\mathrm{X}$ ') of the crossed thermal-guide structural model, and the reference temperatures, used to discriminate between logical output signals, were set to be $370 \mathrm{~K}$ for the prespike-after-postspike pulses and 330K for the prespike-before-postspike pulses, respectively. The change in temperature is given by the difference between the peak intersection temperature for each spike-timing delay and the reference temperature. 
ToC Figure:
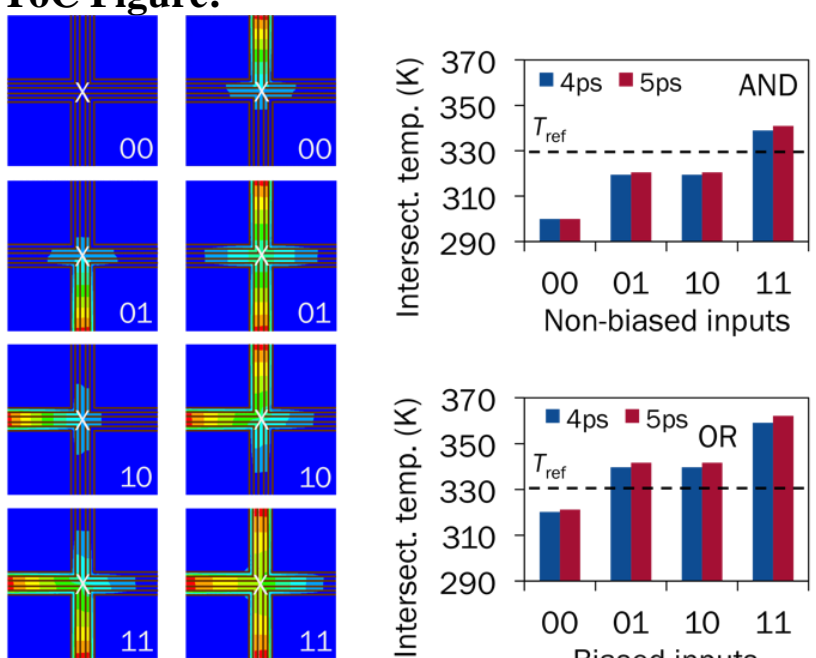

Non-biased
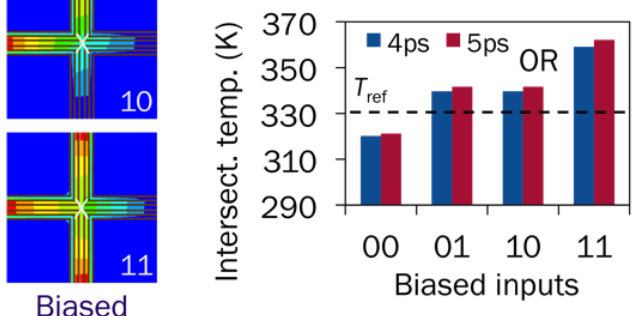